\title{
On Bayesian Analysis of Burr Type VII Distribution under Different Censoring Schemes
}

\author{
Navid Feroze ${ }^{1}$ and Muhammad Aslam² \\ ${ }^{1}$ Department of Mathematics and Statistics, AIOU, Islamabad 44000, Pakistan \\ ${ }^{2}$ Department of Statistics, Quaid-i-Azam University, Islamabad 44000, Pakistan \\ Correspondence should be addressed to Navid Feroze, navidferoz@hotmail.com
}

Received 25 July 2012; Accepted 30 October 2012

Academic Editor: Hongzhong Huang

Copyright (C) 2012 N. Feroze and M. Aslam. This is an open access article distributed under the Creative Commons Attribution License, which permits unrestricted use, distribution, and reproduction in any medium, provided the original work is properly cited.

\begin{abstract}
This paper includes the Bayesian analysis of Burr type VII distribution. Three censoring schemes, namely, left censoring, singly type II censoring, and doubly type II censoring have been used for posterior estimation. The results of different censoring schemes have been compared with those under complete samples. The comparative study among the performance of different censoring schemes has also been made. Two noninformative (uniform and Jeffreys) priors have been assumed to derive the posterior distributions under each case. The performance of Bayes estimators has been compared in terms of posterior risks under a simulation study.
\end{abstract}

\section{Introduction}

Burr [1] introduced twelve forms of the Burr distribution. However, most of the authors have considered the estimation of Burr type XII distribution. The Burr type VII distribution has rarely received any attention. Wahed [2] presented Bayes estimators for the parameters of Burr type XII distribution under the symmetric squared error loss function and the asymmetric linear exponential loss function based on a simple prior distribution. As the estimator turns out to be ratios of integrals, different approximation techniques have been used to obtain approximate Bayes estimators. Real life example has been used to demonstrate the application of Burr type XII distribution. Dasgupta [3] discussed that under certain conditions, the distribution of Burr can be shown to follow an extreme value distribution. Hence, a result on extremal process based on stationary sequence has been proved. Some data sets have been analyzed, and applications of the results have been indicated. Makhdoom and Jafari [4] obtained Bayesian estimators for the shape parameter of the Burr Type XII distribution using grouped and ungrouped data and also consider relationship between them. Bayes point and interval estimators have been derived. Squared error and precautionary loss functions have been considered for the posterior analysis. Monte Carlo simulation has been used to compare the performance of different estimators. Panahi and Asadi [5] considered the statistical inferences based on a Type-II hybrid censored sample from a Burr type XII distribution. As the maximum likelihood estimators cannot be obtained in closed form, a simple fixed point type algorithm has been proposed to compute the maximum likelihood estimators. The approximate confidence intervals for the parameters based on the s-normal approximation to the asymptotic distribution of MLE have been constructed. Bayes estimates of the unknown parameters have also been obtained under the Linex loss function using two approximations. Monte Carlo simulations have been performed to observe the behavior of the proposed methods.

The probability density function of Burr type VII distribution is

$$
\begin{array}{r}
f(x)=\theta 2^{-\theta} \operatorname{sech}^{2}(x)\{1+\tanh (x)\}^{\theta-1}, \\
-\infty<x<\infty, \theta>0 .
\end{array}
$$

And the cumulative distribution function of the distribution 
is

$$
F(x)=2^{-\theta}\{1+\tanh (x)\}^{\theta} .
$$

The probability density function and cumulative distribution function of the Burr type VII distribution can be, respectively, presented as

$$
\begin{gathered}
f(x)=\theta \operatorname{sech}^{2}(x) e^{-\ln \{1+\tanh (x)\}} e^{-\theta \ln \{(1+\tanh (x)) / 2\}^{-1}}, \\
F(x)=e^{-\theta \ln \{(1+\tanh (x)) / 2\}^{-1}} .
\end{gathered}
$$

Under inverse transformation method of simulation the random numbers can be generated from the distribution by using the following formula, where $U$ is uniformly distributed random variable:

$$
x=\tanh ^{-1}\left\{\left(\frac{u}{2^{-\theta}}\right)^{1 / \theta}-1\right\}
$$

where $\tanh ^{-1}(x)=(1 / 2) \ln ((1+x) /(1-x))$.

\section{Posterior Analysis under Complete Data}

In this section, the Bayes estimators along with posterior risks have been derived under the assumption of uniform and Jeffreys priors using complete data. Squared error loss function (SELF) and precautionary loss function (PLF) have been used for estimation. In order to derive the Bayes estimators and corresponding risks, the first step is to obtain the likelihood function. The likelihood function for a sample of size " $n$ " observation is

$$
L(\theta \mid \underline{x}) \propto \theta^{n} e^{-\theta T}
$$

where $T=\sum_{i=1}^{n} \ln \left\{\left(1+\tanh \left(x_{i}\right)\right) / 2\right\}^{-1}$.

The uniform prior is assumed to be

$$
p(\theta) \propto 1 ; \quad \theta>0
$$

The posterior distribution under the assumption of uniform prior is

$$
p(\theta \mid \underline{x})=\frac{[T]^{n+1}}{\Gamma(n+1)} \theta^{n} e^{-\theta T}, \quad \theta>0 .
$$

The Bayes estimators and corresponding posterior risks under squared error loss function (SELF) can be derived by using the following formulae:

$$
\begin{gathered}
\theta_{\text {SELF }}=E(\theta \mid \underline{x}), \\
\rho\left(\theta_{\text {SELF }}\right)=E\left(\theta^{2} \mid \underline{x}\right)-\{E(\theta \mid \underline{x})\}^{2} .
\end{gathered}
$$

Bayes estimator and posterior risk under uniform prior using SELF are

$$
\theta_{\mathrm{SELF}}=\frac{n+1}{T}, \quad \rho\left(\theta_{\mathrm{SELF}}\right)=\frac{n+1}{T^{2}} .
$$

Similarly, The Bayes estimators and corresponding posterior risks under precautionary loss function (PLF) can be derived by using the following formulae:

$$
\begin{gathered}
\theta_{\mathrm{PLF}}=\left\{E\left(\theta^{2} \mid \underline{x}\right)\right\}^{1 / 2}, \\
\rho\left(\theta_{\mathrm{PLF}}\right)=2\left[\left\{E\left(\theta^{2} \mid \underline{x}\right)\right\}^{1 / 2}-E(\theta \mid \underline{x})\right] .
\end{gathered}
$$

The derivations of Bayes estimators and associated posterior risks using precautionary loss function have not been presented in the paper. These can easily be derived from the concerned posterior distributions.

The Jeffreys prior has been derived to be

$$
p(\theta) \propto \frac{1}{\theta}, \quad \theta>0
$$

The posterior distribution under the assumption of Jeffreys prior is

$$
p(\theta \mid \underline{x})=\frac{T^{n}}{\Gamma(n)} \theta^{n-1} e^{-\theta T}, \quad \theta>0
$$

Bayes estimator and risk under Jeffreys prior using SELF are

$$
\theta_{\mathrm{SELF}}=\frac{n}{T}, \quad \rho\left(\theta_{\mathrm{SELF}}\right)=\frac{n}{T^{2}}
$$

\section{Posterior Analysis under Left Censored Samples}

Let $X_{r+1}, \ldots, X_{n}$ be last $n-r$ order statistics from a sample of size $n$ from Burr type VII distribution. Then the likelihood function for the $X_{r+1}, \ldots, X_{n}$ observations is

$$
\begin{gathered}
L(\theta \mid \underline{x}) \propto\left\{F\left(x_{r+1}\right)\right\}^{r} \prod_{i=r+1}^{n} f\left(x_{i}\right), \\
L(\theta \mid \underline{x}) \propto \theta^{n-r} e^{-\theta \xi_{1 r}},
\end{gathered}
$$

where

$$
\xi_{1 r}=r \ln \left\{\frac{1+\tanh \left(x_{r+1}\right)}{2}\right\}^{-1}+\sum_{i=r+1}^{n} \ln \left\{\frac{1+\tanh \left(x_{i}\right)}{2}\right\}^{-1} .
$$

The posterior distribution under uniform prior is

$$
p(\theta \mid \underline{x})=\frac{\left(\xi_{1 r}\right)^{n-r+1}}{\Gamma(n-r+1)} \theta^{n-r} e^{-\theta \xi_{1 r}} .
$$


The Bayes estimator and posterior risk under uniform prior using SELF are

$$
\theta_{\mathrm{SELF}}=\frac{n-r+1}{\xi_{1 r}}, \quad \rho\left(\theta_{\mathrm{SELF}}\right)=\frac{n-r+1}{\left(\xi_{1 r}\right)^{2}}
$$

The posterior distribution under Jeffreys prior is

$$
p(\theta \mid \underline{x})=\frac{\left(\xi_{1 r}\right)^{n-r}}{\Gamma(n-r)} \theta^{n-r-1} e^{-\theta \xi_{1 r}} .
$$

The Bayes estimator and posterior risk under Jeffreys prior using SELF are

$$
\theta_{\mathrm{SELF}}=\frac{n-r}{\xi_{1 r}}, \quad \rho\left(\theta_{\mathrm{SELF}}\right)=\frac{n-r}{\left(\xi_{1 r}\right)^{2}} .
$$

\section{Posterior Analysis under Singly Type II Censored Samples}

Suppose " $n$ " items are put on a life-testing experiment, and only first " $m$ " failure times have been observed, that is, $x_{1}<x_{2} \cdots<x_{m}$ and remaining " $n-m$ " items are still working. Under the assumptions that the lifetimes of the items are independently and identically distributed Burr type VII random variable, the likelihood function of the observed data, is

$$
\begin{aligned}
& L(\theta \mid \underline{x}) \propto\left[\prod_{i=1}^{m} f\left(x_{i}\right)\right]\left[1-F\left(x_{m}\right)\right]^{n-m}, \\
& L(\theta \mid \underline{x}) \propto \sum_{j=0}^{n-m}(-1)^{j}\left(\begin{array}{c}
n-m \\
j
\end{array}\right) \theta^{m} e^{-\theta \psi_{1 j}},
\end{aligned}
$$

where

$$
\psi_{1 j}=\sum_{i=1}^{m} \ln \left\{\frac{1+\tanh \left(x_{i}\right)}{2}\right\}^{-1}+j \ln \left\{\frac{1+\tanh \left(x_{m}\right)}{2}\right\}^{-1} .
$$

The posterior distribution under uniform prior is

$$
p(\theta \mid \underline{x})=\frac{1}{C_{1}} \sum_{j=0}^{n-m}(-1)^{j}\left(\begin{array}{c}
n-m \\
j
\end{array}\right) \theta^{m} e^{-\theta \psi_{1 j}},
$$

where $C_{1}=\sum_{j=0}^{n-m}(-1)^{j}\left(\begin{array}{c}n-m \\ j\end{array}\right)\left(\Gamma(m+1) /\left(\psi_{1 j}\right)^{m+1}\right)$.

The Bayes estimator and posterior risk under uniform prior using SELF are

$$
\begin{aligned}
\theta_{\text {SELF }}= & \frac{1}{C_{1}} \sum_{j=0}^{n-m}(-1)^{j}\left(\begin{array}{c}
n-m \\
j
\end{array}\right) \frac{\Gamma(m+2)}{\left(\psi_{1 j}\right)^{m+2}}, \\
\rho\left(\theta_{\text {SELF }}\right)= & \frac{1}{C_{1}} \sum_{j=0}^{n-m}(-1)^{j}\left(\begin{array}{c}
n-m \\
j
\end{array}\right) \frac{\Gamma(m+3)}{\left(\psi_{1 j}\right)^{m+3}} \\
& -\left\{\frac{1}{C_{1}} \sum_{j=0}^{n-m}(-1)^{j}\left(\begin{array}{c}
n-m \\
j
\end{array}\right) \frac{\Gamma(m+2)}{\left(\psi_{1 j}\right)^{m+2}}\right\}^{2} .
\end{aligned}
$$

The posterior distribution under Jeffreys prior is

$$
p(\theta \mid \underline{x})=\frac{1}{C_{2}} \sum_{j=0}^{n-m}(-1)^{j}\left(\begin{array}{c}
n-m \\
j
\end{array}\right) \theta^{m-1} e^{-\theta \psi_{1 j}},
$$

where $C_{2}=\sum_{j=0}^{n-m}(-1)^{j}\left(\begin{array}{c}n-m \\ j\end{array}\right)\left(\Gamma(m) /\left(\psi_{1 j}\right)^{m}\right)$.

The Bayes estimator and posterior risk under Jeffreys prior using SELF are

$$
\begin{aligned}
\theta_{\text {SELF }} & =\frac{1}{C_{1}} \sum_{j=0}^{n-m}(-1)^{j}\left(\begin{array}{c}
n-m \\
j
\end{array}\right) \frac{\Gamma(m+1)}{\left(\psi_{1 j}\right)^{m+1}}, \\
\rho\left(\theta_{\text {SELF }}\right)= & \frac{1}{C_{1}} \sum_{j=0}^{n-m}(-1)^{j}\left(\begin{array}{c}
n-m \\
j
\end{array}\right) \frac{\Gamma(m+2)}{\left(\psi_{1 j}\right)^{m+2}} \\
& -\left\{\frac{1}{C_{1}} \sum_{j=0}^{n-m}(-1)^{j}\left(\begin{array}{c}
n-m \\
j
\end{array}\right) \frac{\Gamma(m+1)}{\left(\psi_{1 j}\right)^{m+1}}\right\}^{2} .
\end{aligned}
$$

\section{Posterior Analysis under Doubly Type II Censored Samples}

Consider a random sample of size " $n$ " from an Burr type $X$ distribution, and let $x_{r}, \ldots, x_{s}$ be the ordered observations remaining when the " $r-1$ " smallest observations and the " $n-s$ " largest observations have been censored. The likelihood function for $\theta$ given the type II doubly censored sample $\underline{x}=\left(x_{r}, \ldots, x_{s}\right)$ is

$$
\begin{gathered}
L(\theta \mid \underline{x}) \propto\left[F\left(x_{r} \mid \theta\right)\right]^{r-1}\left[1-F\left(x_{s} \mid \theta\right)\right]^{n-s} \prod_{i=r}^{s} f\left(x_{i} \mid \theta\right), \\
L(\theta \mid \underline{x}) \propto \sum_{j=0}^{n-s}(-1)^{j}\left(\begin{array}{c}
n-s \\
j
\end{array}\right) \theta^{k} e^{-\theta \psi_{2 j}}, \\
\psi_{2 j}=(r-1) \ln \left\{\frac{1+\tanh \left(x_{r}\right)}{2}\right\}^{-1} \\
+\sum_{i=r}^{s} \ln \left\{\frac{1+\tanh \left(x_{i}\right)}{2}\right\}^{-1}+j \ln \left\{\frac{1+\tanh \left(x_{s}\right)}{2}\right\}^{-1},
\end{gathered}
$$

and $k=s-r+1$. 
The posterior distribution under uniform prior is

$$
p(\theta \mid \underline{x})=\frac{1}{C_{3}} \sum_{j=0}^{n-s}(-1)^{j}\left(\begin{array}{c}
n-s \\
j
\end{array}\right) \theta^{k} e^{-\theta \psi_{2 j}},
$$

where $C_{3}=\sum_{j=0}^{n-s}(-1)^{j}\left(\begin{array}{c}n-s \\ j\end{array}\right)\left(\Gamma(k+1) /\left(\psi_{2 j}\right)^{k+1}\right)$.

The Bayes estimator and posterior risk under uniform prior using SELF are

$$
\begin{aligned}
\theta_{\mathrm{SELF}} & =\frac{1}{C_{3}} \sum_{j=0}^{n-s}(-1)^{j}\left(\begin{array}{c}
n-s \\
j
\end{array}\right) \frac{\Gamma(k+2)}{\left(\psi_{2 j}\right)^{k+2}}, \\
\rho\left(\theta_{\mathrm{SELF}}\right)= & \frac{1}{C_{3}} \sum_{j=0}^{n-s}(-1)^{j}\left(\begin{array}{c}
n-s \\
j
\end{array}\right) \frac{\Gamma(k+3)}{\left(\psi_{2 j}\right)^{k+3}} \\
& -\left\{\frac{1}{C_{3}} \sum_{j=0}^{n-s}(-1)^{j}\left(\begin{array}{c}
n-s \\
j
\end{array}\right) \frac{\Gamma(k+2)}{\left(\psi_{2 j}\right)^{k+2}}\right\}^{2} .
\end{aligned}
$$

The posterior distribution under Jeffreys prior is

$$
p(\theta \mid \underline{x})=\frac{1}{C_{4}} \sum_{j=0}^{n-s}(-1)^{j}\left(\begin{array}{c}
n-s \\
j
\end{array}\right) \theta^{k-1} e^{-\theta \psi_{2 j}},
$$

where $C_{4}=\sum_{j=0}^{n-s}(-1)^{j}\left(\begin{array}{c}n-s \\ j\end{array}\right)\left(\Gamma(k) /\left(\psi_{2 j}\right)^{k}\right)$.

The Bayes estimator and posterior risk under Jeffreys prior using SELF are

$$
\begin{gathered}
\theta_{\mathrm{SELF}}=\frac{1}{C_{4}} \sum_{j=0}^{n-s}(-1)^{j}\left(\begin{array}{c}
n-s \\
j
\end{array}\right) \frac{\Gamma(k+1)}{\left(\psi_{2 j}\right)^{k+1}}, \\
\rho\left(\theta_{\mathrm{SELF}}\right)=\frac{1}{C_{4}} \sum_{j=0}^{n-s}(-1)^{j}\left(\begin{array}{c}
n-s \\
j
\end{array}\right) \frac{\Gamma(k+2)}{\left(\psi_{2 j}\right)^{k+2}} \\
-\left\{\frac{1}{C_{4}} \sum_{j=0}^{n-s}(-1)^{j}\left(\begin{array}{c}
n-s \\
j
\end{array}\right) \frac{\Gamma(k+1)}{\left(\psi_{2 j}\right)^{k+1}}\right\}^{2} .
\end{gathered}
$$

\section{Simulation Study}

Simulation study is a useful technique to assess and compare the performance of different estimators numerically. A simulation study often reflects the patterns of the real life data. Here, the inverse transformation technique of simulation has been used for $n=50,70,100$, and 150 under the parametric space $\theta \in(3,6)$. As a single sample cannot fully describe the behaviors and properties of the estimators, the samples have been replicated 1000 times, and an average of the results has been presented. The magnitude of posterior risks, associated with each estimator, has been underlined in the tables.
TABLE 1: Bayes estimators and risks under uniform prior using SELF.

\begin{tabular}{lcccc}
\hline \multirow{2}{*}{ Sample size } & \multicolumn{4}{c}{ Uniform prior $(\theta=3)$} \\
& CD & LC & S2C & D2C \\
\hline \multirow{2}{*}{50} & 3.0503 & 3.2268 & 3.3793 & 2.9098 \\
\multirow{2}{*}{70} & $\underline{0.1824}$ & $\underline{0.1829}$ & $\underline{0.2278}$ & $\underline{0.1852}$ \\
\multirow{2}{*}{100} & 3.0108 & 3.1851 & 3.3382 & 2.8617 \\
& $\underline{0.1277}$ & $\underline{0.1280}$ & $\underline{0.1596}$ & $\underline{0.1288}$ \\
\multirow{2}{*}{150} & 3.0388 & 3.2147 & 3.3712 & 2.8854 \\
& $\underline{0.0914}$ & $\underline{0.0917}$ & $\underline{0.1144}$ & $\underline{0.0921}$ \\
\hline
\end{tabular}

CD: complete data, LC: left censored data, S2C: singly type II censored data, D2C: doubly type II censored data.

TABle 2: Bayes estimators and risks under uniform prior using PLF.

\begin{tabular}{lcccc}
\hline \multirow{2}{*}{ Sample size } & \multicolumn{4}{c}{ Uniform prior $(\theta=3)$} \\
& CD & LC & S2C & D2C \\
\hline \multirow{2}{*}{50} & 3.0800 & 3.2583 & 3.4128 & 2.9414 \\
\multirow{2}{*}{70} & $\underline{0.0595}$ & $\underline{0.0630}$ & $\underline{0.0671}$ & $\underline{0.0633}$ \\
\multirow{2}{*}{100} & 3.0319 & 3.2074 & 3.3620 & 2.8841 \\
& $\underline{0.0423}$ & $\underline{0.0447}$ & $\underline{0.0476}$ & $\underline{0.0448}$ \\
\multirow{2}{*}{150} & $\underline{3.0538}$ & 3.2305 & 3.3881 & 2.9013 \\
& $\underline{0.0300}$ & $\underline{0.0318}$ & $\underline{0.0339}$ & $\underline{0.0318}$ \\
\hline
\end{tabular}

CD: complete data, LC: left censored data, S2C: singly type II censored data, D2C: doubly type II censored data.

TABLE 3: Bayes estimators and risks under Jeffreys prior using SELF.

\begin{tabular}{lcccc}
\hline \multirow{2}{*}{ Sample size } & \multicolumn{4}{c}{ Jeffreys prior $(\theta=3)$} \\
& CD & LC & S2C & D2C \\
\hline \multirow{2}{*}{50} & 2.9905 & 3.1636 & 3.3119 & 2.8461 \\
\multirow{2}{*}{70} & $\underline{0.1789}$ & $\underline{0.1793}$ & $\underline{0.2231}$ & $\underline{0.1811}$ \\
\multirow{2}{*}{100} & 2.9684 & 3.1402 & 3.2904 & 2.8167 \\
& $\underline{0.1259}$ & $\underline{0.1262}$ & $\underline{0.1572}$ & $\underline{0.1267}$ \\
\multirow{2}{*}{150} & 3.0087 & 3.1828 & 3.3372 & 2.8535 \\
& $\underline{0.0905}$ & $\underline{0.0908}$ & $\underline{0.1132}$ & $\underline{0.0910}$ \\
& $\underline{2.9883}$ & 3.1613 & 3.3037 & 2.8303 \\
\hline
\end{tabular}

CD: complete data, LC: left censored data, S2C: singly type II censored data, D2C: doubly type II censored data.

TABLE 4: Bayes estimators and risks under Jeffreys prior using PLF.

\begin{tabular}{lcccc}
\hline \multirow{2}{*}{ Sample size } & \multicolumn{4}{c}{ Jeffreys prior $(\theta=3)$} \\
& CD & LC & S2C & D2C \\
\hline \multirow{2}{*}{50} & 3.0800 & 3.2583 & 3.3454 & 2.8778 \\
\multirow{2}{*}{70} & $\underline{0.1791}$ & $\underline{0.1895}$ & $\underline{0.0670}$ & $\underline{0.0633}$ \\
\multirow{2}{*}{100} & 3.0319 & 3.2074 & 3.3142 & 2.8391 \\
& $\underline{0.1271}$ & $\underline{0.1344}$ & $\underline{0.0476}$ & $\underline{0.0448}$ \\
\multirow{2}{*}{150} & 3.0538 & 3.2305 & 3.3542 & 2.8694 \\
& $\underline{0.0902}$ & $\underline{0.0954}$ & $\underline{0.0338}$ & $\underline{0.0318}$ \\
\hline
\end{tabular}

CD: complete data, LC: left censored data, S2C: singly type II censored data, D2C: doubly type II censored data. 
TABLE 5: Bayes estimators and risks under uniform prior using SELF.

\begin{tabular}{lcccc}
\hline \multirow{2}{*}{ Sample size } & \multicolumn{4}{c}{ Uniform prior $(\theta=6)$} \\
\hline \multirow{2}{*}{50} & CD & LC & S2C & D2C \\
\multirow{2}{*}{70} & 5.9775 & 6.3235 & 6.6528 & 5.6995 \\
& $\underline{0.7006}$ & $\underline{0.7025}$ & $\underline{0.8830}$ & $\underline{0.7109}$ \\
100 & 5.9814 & 6.3276 & 6.6728 & 5.7147 \\
& $\underline{0.5039}$ & $\underline{0.5053}$ & $\underline{0.6381}$ & $\underline{0.5138}$ \\
150 & 6.0442 & 6.3940 & 6.6872 & 5.7463 \\
& $\underline{0.3617}$ & $\underline{0.3627}$ & $\underline{0.4501}$ & $\underline{0.3651}$ \\
\hline
\end{tabular}

CD: complete data, LC: left censored data, S2C: singly type II censored data, D2C: doubly type II censored data.

TABLE 6: Bayes estimators and risks under uniform prior using PLF.

\begin{tabular}{lcccc}
\hline \multirow{2}{*}{ Sample size } & \multicolumn{4}{c}{ Uniform $\operatorname{prior}(\theta=6)$} \\
& CD & LC & S2C & D2C \\
\hline \multirow{2}{*}{50} & 6.0358 & 6.3852 & 6.7189 & 5.7615 \\
\multirow{2}{*}{70} & $\underline{0.1166}$ & $\underline{0.1234}$ & $\underline{0.1321}$ & $\underline{0.1241}$ \\
& 6.0233 & 6.3720 & 6.7205 & 5.7595 \\
100 & $\underline{0.0839}$ & $\underline{0.0888}$ & $\underline{0.0953}$ & $\underline{0.0896}$ \\
& 6.0740 & 6.4256 & 6.7208 & 5.7780 \\
150 & $\underline{0.0597}$ & $\underline{0.0632}$ & $\underline{0.0671}$ & $\underline{0.0634}$ \\
& 6.0353 & 6.3847 & 6.6764 & 5.7380 \\
& $\underline{0.0398}$ & $\underline{0.0421}$ & $\underline{0.0447}$ & $\underline{0.0422}$ \\
\hline
\end{tabular}

CD: complete data, LC: left censored data, S2C: singly type II censored data, D2C: doubly type II censored data.

TABLE 7: Bayes estimators and risks under Jeffreys prior using SELF.

\begin{tabular}{lcccc}
\hline \multirow{2}{*}{ Sample size } & \multicolumn{4}{c}{ Jeffreys prior $(\theta=6)$} \\
\hline \multirow{2}{*}{50} & $\mathrm{CD}$ & LC & S2C & D2C \\
\multirow{2}{*}{70} & 5.8603 & 6.1995 & 6.5202 & 5.5748 \\
& $\underline{0.6869}$ & $\underline{0.6887}$ & $\underline{0.8648}$ & $\underline{0.6952}$ \\
100 & 5.8971 & 6.2385 & 6.5773 & 5.6249 \\
& $\underline{0.4968}$ & $\underline{0.4981}$ & $\underline{0.6286}$ & $\underline{0.5056}$ \\
150 & 5.9843 & 6.3307 & 6.6200 & 5.6828 \\
& $\underline{0.3581}$ & $\underline{0.3591}$ & $\underline{0.4454}$ & $\underline{0.3611}$ \\
\hline
\end{tabular}

CD: complete data, LC: left censored data, S2C: singly type II censored data, D2C: doubly type II censored data.

TABLE 8: Bayes estimators and risks under Jeffreys prior using PLF.

\begin{tabular}{lcccc}
\hline \multirow{2}{*}{ Sample size } & \multicolumn{4}{c}{ Jeffreys prior $(\theta=6)$} \\
\hline \multirow{2}{*}{50} & CD & LC & S2C & D2C \\
\multirow{2}{*}{70} & 6.0358 & 6.3852 & 6.5862 & 5.6368 \\
& $\underline{0.3510}$ & $\underline{0.3714}$ & $\underline{0.1320}$ & $\underline{0.1240}$ \\
100 & 6.0233 & 6.3720 & 6.6249 & 5.6696 \\
& $\underline{0.2524}$ & $\underline{0.2671}$ & $\underline{0.0952}$ & $\underline{0.0895}$ \\
150 & 6.0740 & 6.4256 & 6.6535 & 5.7145 \\
& $\underline{0.1794}$ & $\underline{0.1898}$ & $\underline{0.0671}$ & $\underline{0.0634}$ \\
\hline
\end{tabular}

CD: complete data, LC: left censored data, S2C: singly type II censored data, D2C: doubly type II censored data.
The results of simulation study, presented in Tables 18 , are obtained under complete and $20 \%$ censored samples. It is immediate from the above study that the magnitude of risk decreases with the increase in the sample size irrespective of nature (complete and censored) of the samples. The estimated value of the parameter converges to the true value very rapidly under complete data; however, in case of censored data the convergence is not that good. While, increase in the true parametric value and the censoring rate imposes a negative impact on the convergence and performance of the estimates. It can also be observed that the performance, in terms of convergence and posterior risk, of precautionary loss function is better than squared error loss function. It is because that the posterior distributions are asymmetric. It can also be assessed that the magnitudes of risks associated with estimates under Jeffreys prior are lesser as compared to those under uniform prior. In comparison of censoring schemes it is found that the left censored samples provide the best results. The estimates under doubly censored samples stand at second position performance wise.

\section{Conclusions and Recommendations}

From the findings of simulation study it can be concluded that in order to estimate the parameter of Burr type VII distribution under a Bayesian framework using censored data, the use of Jeffreys prior, precautionary loss function, and left censored samples can be preferred. The study can further be extended by using some other loss functions; informative priors and mixture of two are more components of Burr type VII distribution. The work on the mixture of two components of Burr type VII distribution is continued.

\section{References}

[1] W. I. Burr, "Cumulative frequency distribution," Annals of Mathematical Statistics, vol. 13, pp. 215-232, 1942.

[2] A. S. Wahed, "Bayesian inference using Burr model under asymmetric loss function: an application to Carcinoma survival data," Journal of Statistical Research, vol. 40, no. 1, pp. 45-57, 2006.

[3] R. Dasgupta, "On the distribution of burr with applications," Sankhya B, vol. 73, pp. 1-19, 2011.

[4] I. Makhdoom and A. Jafari, "Bayesian estimations on the Burr type XII distribution using grouped and un-grouped data," Australian Journal of Basic and Applied Sciences, vol. 5, no. 6, pp. 1525-1531, 2011.

[5] H. Panahi and S. Asadi, "Analysis of the type-II hybrid censored Burr type XII distribution under LINEX loss function," Applied Mathematical Sciences, vol. 5, no. 79, pp. 3929-3942, 2011. 

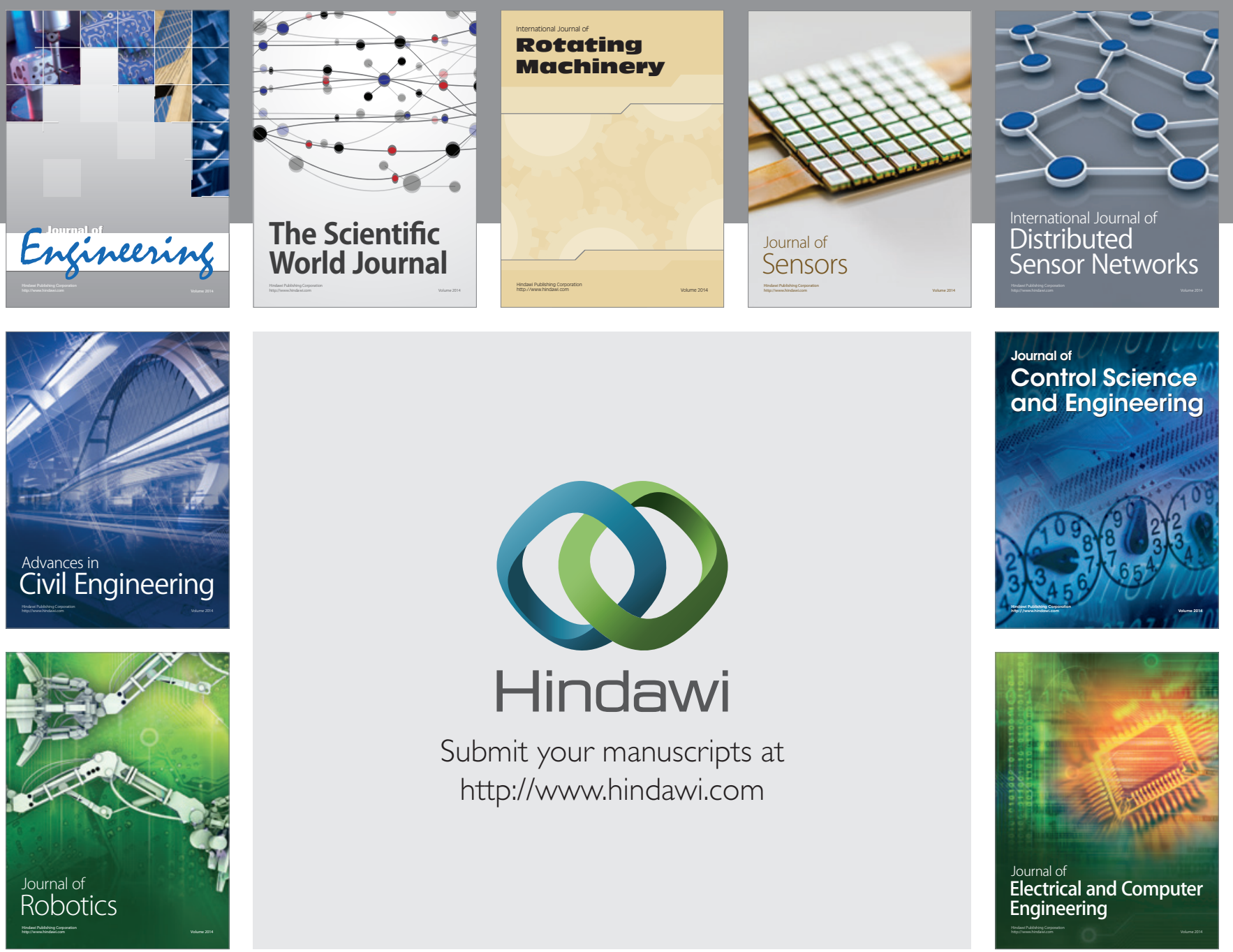

Submit your manuscripts at

http://www.hindawi.com
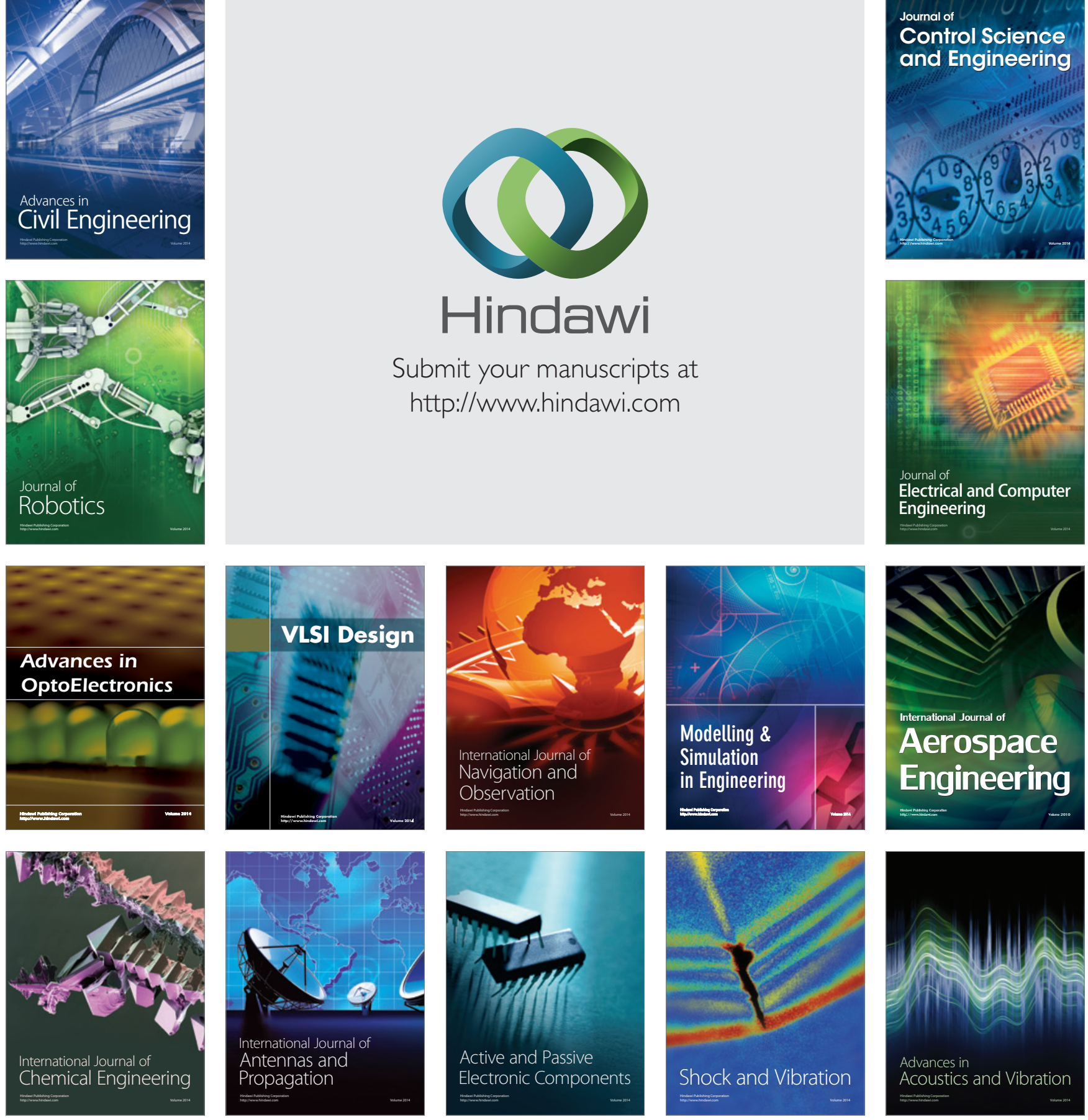